\section{Recurrent syncope in a hypertensive subject with vascular cognitive impair- ment and permanent pacemaker}

\author{
Alberto Mazza, ${ }^{1}$ Roberta Ravenni, 2 \\ Domenico Montemurro, ${ }^{3}$ Gianni Pastore, 4 \\ Laura Schiavon, 1 Domenico Rubello5 \\ 1Department of Internal Medicine and \\ 2Department of Neuroscience, Santa \\ Maria della Misericordia Hospital, Rovigo; \\ 3 Department of Internal Medicine, \\ General Hospital of Vicenza; \\ 4Department of Cardiology and \\ 5Department of Nuclear Medicine, \\ Radiology and, Medical Physics, Santa \\ Maria della Misericodia Hospital, Rovigo, \\ Italy
}

\section{Abstract}

Syncope following permanent pacemaker (PM) implantation is a nightmare for electrophysiologists. We describe a case of daily recurrent syncope in an 84-year-old man having a dual-chamber pacemaker implanted for complete atrio-ventricular block occurred 4 years before the admission to our department. He had a history of arterial hypertension, parossistic atrial fibrillation, chronic obstructive pulmonary disease, stage-III chronic renal failure, mild vascular cognitive impairment and glaucoma. The initial work-up including electrocardiogram (ECG), repeated PM interrogations, Holter electrocardiogram, blood pressure measurement in orthostatic position, complete blood count, serum glycaemia, electrolytes and thyroid function tests showed normal findings. Syncope occurred in lying position and during $90^{\circ}$ left clockwise neck rotation and was associated to pallor, sweating, tonic-clonic seizures and transient self-limited loss of consciousness lasting a few seconds. Electroencephalogram was normal. During continuous ECG monitoring, the right rotation of the head determined a ventricular asystolic pause lasting 9 seconds associated with loss of consciousness. Restoration of sinus rhythm was observed after bringing back the head in axis. The PM interrogation, performed during pacing failure, recorded low impedance of bipolar ventricular lead, suggesting a damage in lead insulation. It is likely that lead movements during clockwise neck rotation produced an intermittent short circuit that prevented sufficient energy delivery to the myocardium with a consequence of sudden loss of capture.

\section{Introduction}

Syncope following permanent pacemaker (PM) implantation is a nightmare for electrophysiologists. ${ }^{1}$ Late complications due to PM malfunctioning are uncommon. Pacing system malfunction can present as output failure (e.g. oversensing, electromagnetic interference, make-break signal) or as capture failure due to battery depletion, lead failure or dislodgement, and increase in pacing threshold. ${ }^{2}$ These conditions can be intermittent or continuous.

In this paper we describe a case of recurrent syncope with progressive neurological impairment in an elderly patient showing repeatedly normal PM interrogation.

\section{Case Report}

An 84-year-old man with history of arterial hypertension, paroxistic atrial fibrillation, chronic obstructive pulmonary disease, stage-III chronic renal failure, mild vascular cognitive impairment and glaucoma was admitted to our department for daily new-onset recurrent syncope. Because of an atrio-ventricular block, he had a dual-chamber PM (Sorin Neway, Milan, Italy) implantation 4 years before admission. He was repeatedly evaluated for syncope in an Emergency Unit, where electrocardiogram (ECG), neurological examination and cerebral computer computed tomography (CT) scan were normal. Battery status and leads parameters including sensing amplitudes and impedances were normal, although an elevated right ventricular pacing threshold was found ( $3 \mathrm{~V}$ at $0.5 \mathrm{~ms}$ ). This threshold had been stable in the last 3 years, however. Chest $\mathrm{x}$-ray showed stable lead placement and intact electrocatheter.

Medical regimen consisted of irbesartan (150 mg daily), flecainide (100 mg daily), a combination of formoterol and inhaled budesonide (160/4.5 g twice a day), allopurinol (300 $\mathrm{mg}$ daily), acetilsalicylic acid (100 mg daily), alprazolam (25 mg daily) and dorzolamide/timolol instilled twice. The patient did not have any prodromic symptoms and did not report any palpitations, dizziness or chest pain before syncope and recovered completely on each occasion without neurological sequelae or transient confusion.

Initial physical examination showed 2/6 systolic ejective murmur in cardiac apex and bilateral carotid murmurs; neurological examination did not reveal any abnormalities. Supine blood pressure was $128 / 78 \mathrm{mmHg}$, without orthostatic hypotension. The initial work-up including ECG, electroencephalogram (EEG), complete blood count, serum glycaemia, electrolytes, thyroid function tests, echocardiography and Holter-ECG monitoring showed nor-
Correspondence: Alberto Mazza, Department of Internal Medicine, Santa Maria della Misericordia Hospital, viale Tre Martiri 140, 45100 Rovigo, Italy.

Tel. +39.0425.394567 - Fax: +39.0425.394157.

E-mail:mazza.alberto@azisanrovigo.it

Key words: synsope, neurology, hypertension, pacemaker.

Conflict of interests: the authors report no potential conflict of interests.

Acknowledgements: we would like to thank Prof Edoardo Casiglia, Department of Medicine, University of Padova, Italy, for his contribution to this paper.

Received for publication: 13 December 2011.

Revision received: 23 July 2012.

Accepted for publication: 30 July 2012 .

This work is licensed under a Creative Commons Attribution NonCommercial 3.0 License (CC BYNC 3.0).

(C) Copyright A. Mazza et al., 2012

Licensee PAGEPress, Italy

Neurology International 2012; 4:e12

doi:10.4081/ni.2012.e12

mal findings. The neurologist did not show anything of relevant.

During the hospitalization the patient suffered of a first episode of syncope occurred in lying position during left clockwise neck rotation. In particular, clockwise head rotation at about $90^{\circ}$ was followed by pallor, sweating, tonic-clonic seizures and a transient, self-limited loss of consciousness lasting a few seconds. The clinical picture disappeared bringing back the head in axis. Twelve-lead surface electrocardiogram and cardiovascular parameters were normal.

Ultrasound of the extracranial vessels showed two hypoechogenic plaques (eccentric $60 \%$ stenosis at the origin of both internal carotid arteries); the vertebral arteries were very thin and flow was difficult to measure, especially in the right vertebral artery. The three-dimensional computed tomographic angiography of the extracranial vessels (Figure 1) showed a 50\% stenosis of right vertebral artery at the confluence with the basilar artery and $60 \%$ stenosis of left vertebral artery with extrinsic compression by hypertrophic C6 spinal transverse process. The purpose of the neck maneuvers was to induce the syncope assuming a compressive effect upon the vertebral artery flow during left clockwise neck rotation. However the neurosurgeon and vascular surgeon excluded the possibility of any surgical treatment or endovascular stenting.

Two days after the first syncope, the patient suffered an episode characterized by tonic- 
clonic seizures of head and upper limbs, sphincter incontinence and a transient cognitive decay. Prodromal symptoms were sweating and a sensation of imminent death, chest pain and severe hypotension. Electrocardiogram and electroencephalogram were normal. Patient was transferred to the cardiac intensive care. During continuous ECG monitoring, the spontaneous right rotation of the head determined a 9 second asystolic pause accompanied by loss of consciousness. Restoration of sinus rhythm was obtained by positioning the head in axis (Figure 2A). Electrocardiographic tracing showed sinus rhythm with complete atrio-ventricular block and pacing artefacts not followed by cardiac electrical activity. Further neck rotation reproduced the asystole due to pacing failure (Figure 2B), a condition that disappeared after stopping this manoeuvre (Figure 2C).

The PM interrogation performed during pacing failure recorded low impedance $(<250 \Omega)$ of bipolar ventricular lead (Medtronic 5092, Medtronic Inc., Minneapolis, USA) suggesting damage in lead insulation. The explanation was that lead movements during neck rotation produced intermittent short circuit preventing sufficient energy delivery to the myocardium with sudden loss of capture. ${ }^{3,4}$ Although unipolar output configuration eliminated the problem, new bipolar steroid-eluting active-fixation lead (Medtronic Capsure-Fix ${ }^{\circledR}$ Novus 5076, Medtronic Inc., Minneapolis, USA) was added in the right side of interventricular septum. Due to battery depletion, the PM was replaced. We preferred to implant a 3-chamber PM because of the complete absence of intrinsic ventricular rhythm: the atrial channel was connected to a standard atrial lead, the new electrocatheter (EC) was connected to right ventricular port and the older lead in the left ventricular port. The older EC (with unipolar configuration) was used as a back-up electrode in case of dislodgement of the new lead. Subsequent PM interrogation showed stable pacing threshold and impedance of the new lead, so that the PM was re-programmed to pace only from standard right ventricular port.

\section{Conclusions}

In conclusion, in this elderly hypertensive patient with PM, recurrent syncope involved multiple potential contributors. In such cases, no evident cause can be identified despite extensive diagnostic evaluation. Despite normality of basal and dynamic electrocardiogram, chest $\mathrm{x}$-ray and repeated PM interrogation, in the presence of recurrent syncope PM malfunction should not be excluded. In these cases a prolonged ECG monitoring and body movement manoeuvres during PM evaluation should be also performed to exclude pacing failure.

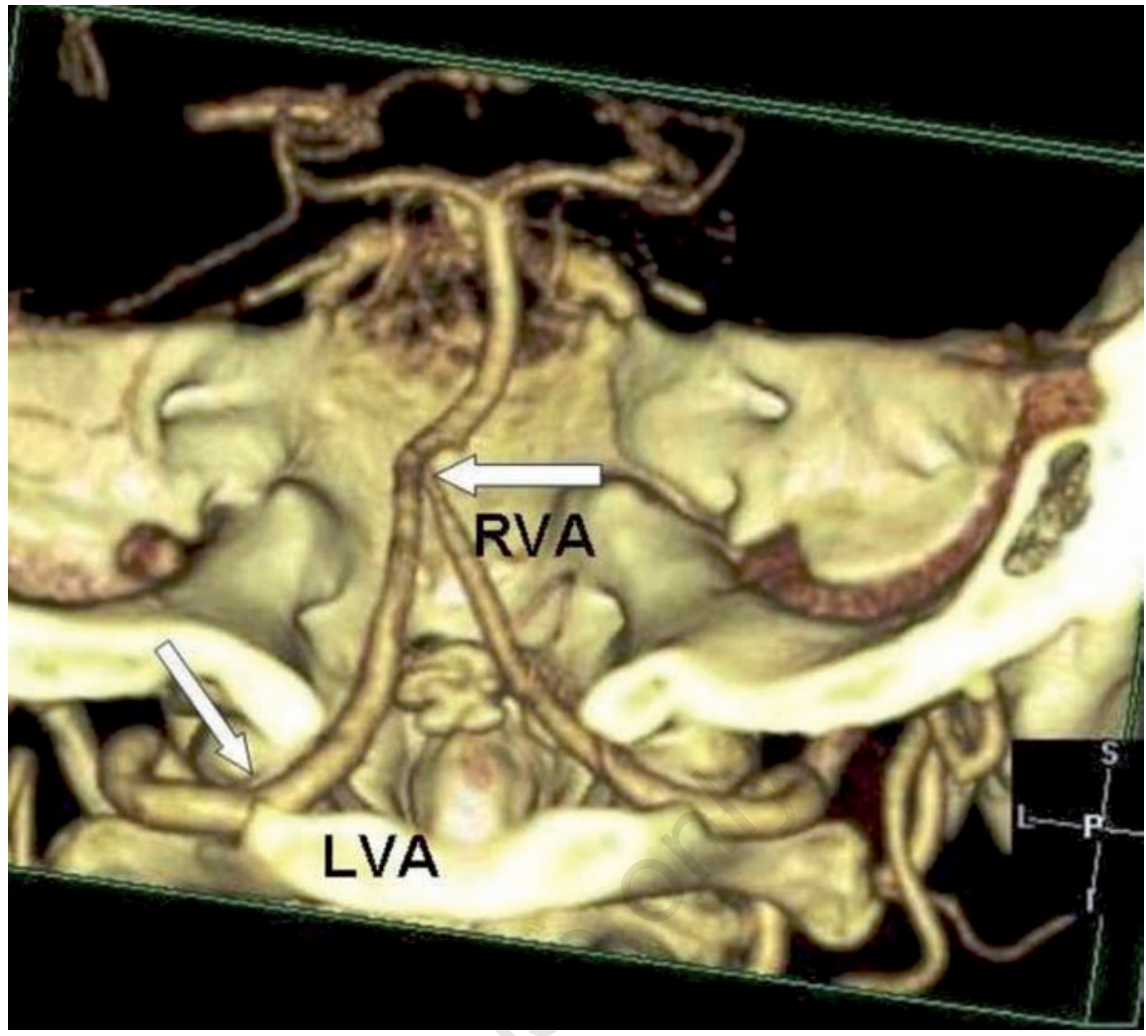

Figure 1. Three-dimensional computed tomographic angiography showing the stenoses (arrows) of the right vertebral artery and left vertebral artery. RVA, right vertebral artery; LVA, left vertebral artery.

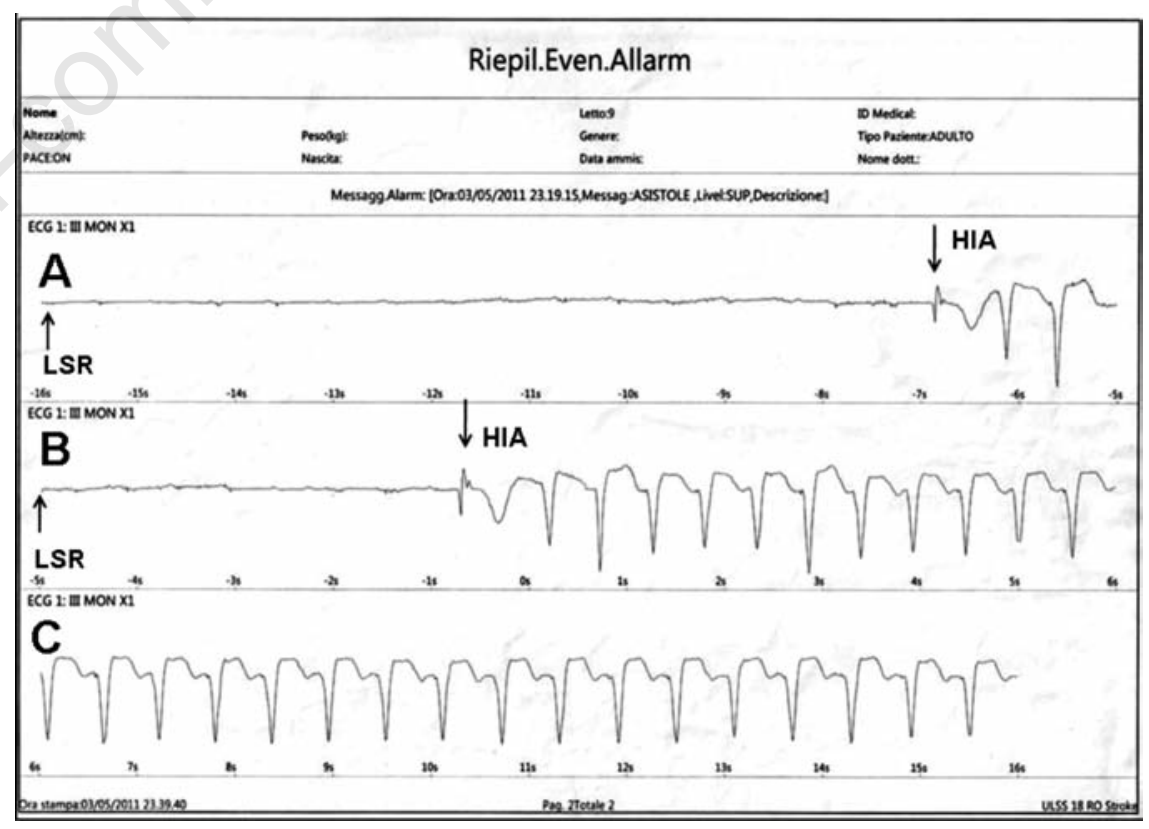

Figure 2. Continuous electrocardiographic monitoring showing sinus rhythm with complete atrio-ventricular block and pacing artefacts not followed by cardiac electrical activity. A systolic pause lasting 9 seconds (A) associated with loss of consciousness follows left side rotation of neck. Restoration of normal pacemaker functioning $(B, C)$ after positioning the head in axis (HIA). LSR, left side rotation, HIA, head in axis. 


\section{References}

1. Levine PA. Differential diagnosis, evaluation and management of pacing system malfunction. In: Ellenbogen $\mathrm{K}$, Wood M, Cardiac pacing. Boston: Blackwell Scientific Publications; 2002. p 345.

2. Mellert F, Esmailzadeh B, Schneider C, et al. An unusual case of pacemaker failure: complete disconnection of connector block and battery of a subpectorally implanted dual chamber pacemaker. Pacing Clin Electrophysiol 2002;25:509-10.

3. Aliyev F, Celiker C, Türko lu C, Abaci 0 . Unusual electrocardiographic presentation of pacemaker battery depletion with lead failure: pacing spike, artefact or native QRS? A short communication. Europace 2008;10:888-9.

4. Antonelli D, Rosenfeld T, Freedberg NA, et al. Insulation lead failure: is it a matter of insulation coating, venous approach, or both? Pacing Clin Electrophysiol 1998;21: 418-21. 